This item was submitted to Loughborough's Research Repository by the author.

Items in Figshare are protected by copyright, with all rights reserved, unless otherwise indicated.

\title{
Chitosan \& conductive PANI/Chitosan composite nanofibers - Evaluation of antibacterial properties
}

PLEASE CITE THE PUBLISHED VERSION

https://doi.org/10.2174/1573413714666181114110651

\section{PUBLISHER}

(c) Bentham Science Publishers Ltd.

\section{VERSION}

AM (Accepted Manuscript)

\section{PUBLISHER STATEMENT}

The published manuscript is available at EurekaSelect via http://www.eurekaselect.com/openurl/content.php? genre=article\&doi=10.2174/1573413714666181114110651.

\section{LICENCE}

CC BY-NC-ND 4.0

\section{REPOSITORY RECORD}

Moutsatsou, Panagiota, Karen Coopman, and Stella Georgiadou. 2019. "Chitosan \& Conductive Pani/chitosan Composite Nanofibers - Evaluation of Antibacterial Properties”. figshare. https://hdl.handle.net/2134/36926. 


\title{
Chitosan \& conductive PANI/Chitosan composite nanofibers - Evaluation of antibacterial properties
}

\author{
Panagiota Moutsatsou, Karen Coopman, Stella Georgiadou * \\ Department of Chemical Engineering, Loughborough University, Loughborough LE11 3TU, Leicestershire, \\ $U K$; \\ * Correspondence: S.Georgiadou@lboro.ac.uk; Tel.: +44-(0)1509-222521
}

\begin{abstract}
Within the healthcare industry, including the treatment of chronic wounds, the challenge of antimicrobial resistance continues to grow. As such, there is a need to develop new treatments that can reduce the bioburden in wounds. The present study is focused on the development of conductive polyaniline (PANI) / chitosan $(\mathrm{CH})$ nanofibrous electrospun membranes and evaluates their antibacterial properties. The blended fibre membranes combine the materials' respective properties such as electrical conductivity, biocompatibility and antibacterial activity. To this end, experimental design was used to determine the electrospinning windows of both pure chitosan and PANI/CH blends of different ratios $(1: 3,3: 5,1: 1)$. The effect of key environmental and process parameters (relative humidity and applied voltage) was determined, as well as the effect of the PANI/CH ratio in the blend, and the molecular interactions between PANI and Chitosan that led to jet stability. The nanofibrous mats were evaluated regarding their morphology and antibacterial effect against gram positive and gram negative bacterial strains, namely $B$. subtilis and E. coli. High PANI content mats show increased bactericidal activity against both bacterial strains. This study suggests that electrospun PANI/CH membranes are promising candidates for healthcare applications such as wound dressings.
\end{abstract}

Keywords: polyaniline, chitosan, nanofibers, wound dressing, antimicrobial properties, electrospinning parameters, voltage, humidity

\section{Introduction}

The wound care sector is one of the largely demanding fields in the pharmaceutical industry. Contributors to this large demand for wound care are those suffering from acute and chronic wounds. Presently, autografting is the standard treatment of complicated wounds (1). However, many patients lack available donor sites and are at high risk of chronic wounds suffering from problematic healing. Polymer nanofibers are introduced to repair tissues as mechanically robust form of material that can be more effective for tissue cell activities than other forms due to the large surface area available (2). Other conventional approaches to wound therapy include primarily various ointments, local antimicrobial agents, and sterile bandages. However, the process of wound healing is a complex one and can be compromised by many contributing factors with the major one being the bacterial infection of the wound. One of the most common methods for treating infections is with antibiotics use. Although antibiotics have been proven to be very useful against wound infections, it has also been reported that their use can be associated with complications such as renal and liver toxicity and the emergence of drug-resistant bacteria is one of this centuries global healthcare challenges (2). This study proposes the use of inherently antibacterial polymeric nanofibers that can be used as a wound dressing, potentially enabling reduced usage of antibiotics.

Polymer nanofibers could be used for the treatment of wounds or burns of a human skin, and they can also be designed for haemostatic devices with some unique characteristics. With the aid of electric field, fine fibers of biodegradable polymers can be either directly sprayed/spun onto the injured location of skin to form a fibrous mat dressing (3) or be prepared in advance and applied on the wound as normal wound dressings. Such nanofibrous dressings are structurally similar to the extracellular matrix (ECM) in tissues which is composed of fibrillar collagen (1). Indeed, a previous study (4) showed that polyaniline/chitosan composite membranes promoted proliferation of human fibroblasts, suggesting that this material combination could let wounds heal by encouraging the formation of normal skin growth and eliminate the formation of scar tissue which would occur in a traditional treatment. Absorptive properties of materials such as chitosan could also remove excess fluid from the wound bed (5). Non-woven nanofibrous membrane mats usually have pore sizes in the range of hundreds of nanometers to $1 \mu \mathrm{m}$, small enough to protect the wound from bacterial penetration via aerosol particle capturing mechanisms. The high surface area of the range of $5-100 \mathrm{~m}^{2} / \mathrm{g}$ that nanofibers typically provide, is extremely efficient for fluid absorption and dermal delivery of growth factors or antimicrobial agents if needed (6). 
Chitosan is the $\mathrm{N}$-deacetylated derivative of chitin, a biopolymer encountered in nature, in many crustaceans and insects as a main component of the exoskeleton. It is a biocompatible material, biodegradable and soluble in slightly acidic aqueous media. It can be easily cast as a film for hydrogel formation or be electrospun with the presence of other polymers. It has also been found to exhibit antibacterial, antifungal, mucoadhesive, immunological, hemostatic (7) and wound healing properties as well as to promote cell adhesion and proliferation (8-10). There has been extensive study on chitosan and its derivatives with respect to its antimicrobial activity against several bacteria (11) and two mechanisms are generally used to explain its antimicrobial activity. Firstly, chitosan is believed to chelate nutrients and minerals that are essential for bacteria growth (12), and secondly, as a cationic polyelectrolyte it is believed to be electrostatically interacting with the bacterial cell's membrane, thus impeding proper cell membrane function, leakage of proteinaceous and other intracellular constituents, cell lysis and death $(11,13)$.

Polyaniline (PANI) on the other hand is a conducting polymer whose conjugative structure allows for electron mobility within the molecule chain and therefore renders it electroactive. It has also been reported in the literature that the conductive state of PANI exhibits an antibacterial effect against both gram negative $E$. coli and gram positive Staphylococcus Aureus (14). However, in that study, the PANI was polymerized straight on the substrate that was used further for the evaluation of its antibacterial properties, without providing any details on the removal of chemical residues that occur during this process. In another study performed by Kucekova et al, the antibacterial effect of both doped (salt) and undoped (base) PANI was studied against the same model microorganisms. It was found that PANI base only had a marginal antibacterial effect against $S$. Aureus and none against E. coli, while PANI salt exhibited significant antibacterial activity against both microorganisms (15). Since the PANI salt is more conductive than the PANI base, again here no definitive conclusion can be drawn as to if the antibacterial effect is due to electrostatic interaction of the positively charged molecule and the negatively charged bacteria's cell wall as it has been proposed by Gizdavic-Nikolaidis et al. (16), which causes eruption of the cell wall, lysis and death. Hence, the debate is still open, as on whether the antimicrobial action observed is a result of the agents, that are used during the processing of these materials, usually toxic and detrimental to bacteria and cells alike (17).

However, the electrospinning (which is the most promising technique for the mass production of nanofibers) of both pure chitosan and pure PANI solutions has proved challenging (18). Chitosan aqueous acidic solutions are very viscous with high surface tension which puts an obstacle to the production of uniform nanofibrous mats. In addition to that, the protonation of its free amino groups that takes place when chitosan is dissolved in acidic media, renders it a polyelectrolyte. Therefore, the repulsive forces between ionic groups within the polymer backbone that arise due to the application of a high electric field during electrospinning, restrict the formation of continuous fibres and hinder the electrospinning process (19). It is only recently that a suitable solvent for the electrospinning of chitosan without the need for a carrier polymer has been identified, namely TFA, but, to our knowledge, there is no systematic study on the electrospinning of chitosan, looking into the parameters affecting this process. It seems that the concentrated acid helps to maintain the surface tension at low levels, while allowing the dilution of chitosan at high enough concentrations so as for the critical entanglement concentration to be reached (20) and the solution to become electrospinnable. Therefore, the first part of this study is focused towards the determination of the electrospinning window of pure chitosan as there is lack of insight in the conditions that render pure chitosan electrospinnable. Regarding PANI, it is its conjugative structure mentioned earlier that limits its solubility and hence its processability into nanofibrous structures. However, the production of composite nanofibres consisting of these two polymers seems to overcome these limitations and to show promise for a variety of applications, as it is expected to combine important properties such as biocompatibility, electroactivity and inherent bactericidal activity. In this study, design of experiments (DoE) is used to determine the electrospinning window of chitosan solutions (as up till now, only trial and error electrospinning of pure chitosan has been reported) and even further for the electrospinnability of PANI/chitosan blend solutions. Furthermore, the effect of different PANI/CH ratios on the electrospinning process and on the antibacterial properties of PANI/CH mats will be assessed.

\section{Materials and Methods}

Polyaniline emeraldine base (PANI, Mw=50000), ammonium persulfate (APS), aniline hydrochloride, and (1R)-10-camphor-sulfonic acid (CSA) were purchased from Sigma Aldrich Inc. Trifluoroacetic acid, 1-Methyl- 
2-pyrrolidinone (NMP), chitosan of Mw: $600000-800000$ and 90\% degree of deacetylation, were purchased from Acros Organics. All materials were used without any further purification.

\subsection{Preparation of electrospinning solutions \& electrospinning process}

The following solutions were prepared for electrospinning: A. 3\% w/v chitosan in TFA:DCM (80:20), B. 5\%w/v chitosan in TFA:DCM and C. 5\% PANI/CH blends (3 different ratios 1:3, 3:5, 1:1) in TFA:DCM (80:20). Based on some preliminary experiments which are not presented here and on the literature, TFA was identified as the only suitable solvent for studying the electrospinnability of these blends, due to its effectiveness as PANI and chitosan common solvent (21). This can be attributed to a more expanded conformation that PANI molecules exhibit when dissolved in TFA, in opposition to most commonly used solvents such as chloroform where a more compact-coil conformation is observed (21). In practice, this eliminates the need for removal of undispersed particles by filtration, even at high PANI concentrations, as it is the case for chloroform, which is currently the most common solvent for electrospinning of PANI based solutions. Also, TFA's properties as found in the literature, e.g. relatively low boiling point: $72.4^{\circ} \mathrm{C}$ and low surface tension: $\sim 13.5 \mathrm{dyn} / \mathrm{cm}$ (22) are expected to be very suitable for electrospinning. DCM was also used as a second solvent at a lower ratio $(20 \%)$ as its high volatility (boiling point: $39.6^{\circ} \mathrm{C}$ has been proved to favor the electrospinnability of chitosan when combined with TFA at a ratio of 80:20 (18). The conductivity of the solutions was measured with a 470 Jenway conductivity meter.

The blends were fed through a plastic syringe to the needle tip (20G diameter) and were electrospun under different voltages, produced by a high voltage source (Glassman High Voltage Inc.). The nanofibres were collected on a flat grounded collector covered with aluminum foil. The needle tip to collector distance was fixed at $12 \mathrm{~cm}$ at a horizontal orientation. The flow rate of the solutions was controlled by a syringe pump (Harvard Apparatus).

In order to control the humidity, the electrospinning setup was modified in house. A second floor was added below the chamber's floor at a distance of around $2 \mathrm{~cm}$. A hole was drilled in the centre of the added floor and was connected to a compressed dry air supply. The space in between the two floors allowed the dry air to build up there and flow towards the edges of the floor, leading in the electrospinning chamber. This way, the dry air flows in the chamber, altering the humidity but without intervening with the electrospun fibres, as it passes only through the two edges, behind the nozzle and behind the collector and moving upwards towards the extraction. With this modification, any evaporating solvent building up around the nozzle was being removed as well. A schematic is presented in the Supplementary figures.

The humidity and temperature in the electrospinning chamber were monitored using a temperature and humidity meter ST-321. The morphology of the electrospun mats was then examined with the use of Carl Zeiss (Leo) Scanning Electron Microscope (Model 1530VP). The electrospun membranes were gold coated under vacuum before scanning electron microscopy. The SEM images presented in the results and discussion section are representative of three electrospinning runs and at least three different areas of each mat. For the determination of the average diameter of the fibres and for the generation of charts and plots, AxioVision and Matlab R2016a software were used respectively. 


\subsection{Design of experiments (DoE) for nanofibrous mat formation}

In order to determine the solution concentrations and the electrospinning conditions that would result in successfully electrospun PANI/Chitosan (PANI/CH) composite mats, a thorough study of the electrospinning window of pure chitosan was necessary, since there is no relevant detailed study in the literature. There are studies reporting the successful electrospinning of chitosan in TFA, but most of them focus on the molecular weight and degree of deacetylation of the chitosan and not on the solution parameters or the electrospinning parameters and how these affect electrospinnability $(18,23)$.

For this reason a two-level three factor full factorial design $\left(2^{3}\right)$ was implemented for the investigation of the electrospinning of chitosan in TFA:DCM (80:20) solvent (Table 1).

Table 1: $2^{3}$ full factorial experimental design for the electrospinnability of chitosan solutions

\begin{tabular}{lll} 
Concentration $(\% \mathrm{w} / \mathrm{v})$ & Relative Humidity $(\%)$ & Voltage $(\mathrm{kV})$ \\
\hline 3 & 50 & 28.5 \\
3 & 50 & 23 \\
3 & 20 & 28.5 \\
3 & 20 & 23 \\
\hline 5 & 50 & 28.5 \\
5 & 50 & 23 \\
5 & 20 & 28.5 \\
5 & 20 & 23 \\
\hline
\end{tabular}

After the effect of the chitosan concentration was evaluated, and one concentration was chosen, further experiments were carried out for the investigation of the combined effect of the PANI ratio, the applied voltage and the humidity on the electrospinnability of PANI/CH blends, by using a mixed level full factorial experimental design as shown in Table 2 .

Table 2: Mixed level factorial experimental design for the electrospinnability of PANI/CH blends

\begin{tabular}{llll} 
Run & PANI:CH ratio & Humidity $(\%)$ & Voltage $(\mathrm{kV})$ \\
\hline 1 & $0: 1$ & 50 & 23 \\
2 & $0: 1$ & 50 & 26 \\
3 & $0: 1$ & 50 & 28,5 \\
\cline { 2 - 3 } 4 & $0: 1$ & 35 & 23 \\
5 & $0: 1$ & 35 & 26 \\
6 & $0: 1$ & 35 & 28,5 \\
7 & $0: 1$ & 20 & 23 \\
8 & $0: 1$ & 20 & 26 \\
9 & $0: 1$ & 20 & 28,5 \\
\hline 10 & $1: 3$ & 50 & 23 \\
11 & $1: 3$ & 50 & 26 \\
12 & $1: 3$ & 50 & 28,5 \\
13 & $1: 3$ & 35 & 23 \\
14 & $1: 3$ & 35 & 26 \\
15 & $1: 3$ & 35 & 28,5 \\
16 & $1: 3$ & 20 & 23 \\
17 & $1: 3$ & 20 & 26 \\
18 & $1: 3$ & 20 & 28,5 \\
\hline 19 & $3: 5$ & 50 & 23 \\
20 & $3: 5$ & 50 & 26 \\
21 & $3: 5$ & 50 & 28,5 \\
22 & $3: 5$ & 35 & 23
\end{tabular}




\begin{tabular}{llll}
23 & $3: 5$ & 35 & 26 \\
24 & $3: 5$ & 35 & 28,5 \\
\cline { 2 - 3 } 25 & $3: 5$ & 20 & 23 \\
26 & $3: 5$ & 20 & 26 \\
27 & $3: 5$ & 20 & 28,5 \\
\hline 28 & $1: 1$ & 50 & 23 \\
29 & $1: 1$ & 50 & 26 \\
30 & $1: 1$ & 50 & 28,5 \\
31 & $1: 1$ & 35 & 23 \\
32 & $1: 1$ & 35 & 26 \\
33 & $1: 1$ & 35 & 28,5 \\
34 & $1: 1$ & 20 & 23 \\
35 & $1: 1$ & 20 & 26 \\
36 & $1: 1$ & 20 & 28,5 \\
\hline
\end{tabular}

For both experimental designs, as can be seen in Tables $1 \& 2$, the maximum and minimum values for each parameter were determined. The chitosan concentration values were chosen based on the literature $(24,25)$. Higher concentration $(7 \% \mathrm{w} / \mathrm{v})$ has also been examined in preliminary experiments but yielded highly viscous solutions that were not suitable for electrospinning. As for the voltage range, the upper limit of $28.5 \mathrm{kV}$ was defined by the technical limitations of the equipment. As low voltage setting, the lowest one allowing Taylor cone formation was used, which would indicate the potential formation of a jet. When it comes to the humidity, as maximum level, the ambient humidity value without dry air flowing inside the chamber was used, and as minimum level, again the lowest humidity value that could be attained by the dry air flow was used.

\subsection{Determination of $\mathrm{CH}$ and $\mathrm{PANI} / \mathrm{CH}$ membrane antibacterial properties}

To assess the effect of protonation on the bactericidal activity of chitosan, and to obtain insight into the main antibacterial mechanism of chitosan, two different methods of chitosan crosslinking were used, one resulting in protonated mat and the other in a deprotonated mat. The antibacterial activities of PANI-Chitosan (PANI/CH) membranes were investigated by a zone inhibition method (26). In order to avoid remaining aniline monomers that could tamper with the antibacterial test, commercial PANI was used in this study and thorough washings of the PANI containing membranes preceded the antibacterial test. Gram-negative Escherichia coli (E. coli strain K12) and gram-positive Bacillus subtilis (B. subtilis) cells were used as the model microorganisms. All electrospun mats with different contents of PANI were cut into $2 \mathrm{~cm}$ diameter discs, thoroughly washed with PBS and ethanol, and sterilized by UV light prior to bacterial viability test. Nutrient agar plates were inoculated with $1 \mathrm{~mL}$ of bacterial suspension containing around $1 \times 10^{5}$ colony forming units (CFUs) $/ \mathrm{mL}$ bacteria. The composite nanofibres were gently placed on the inoculated plates and were incubated at $37^{\circ} \mathrm{C}$ for $24 \mathrm{~h}$ for the incubation of E. coli and at $35^{\circ} \mathrm{C}$ for $24 \mathrm{~h}$ for the B. subtilis. The inhibition zone around each sample was determined by measuring with a ruler the diameter of the inhibition area around each disk, in $\mathrm{mm}$ and subtracting from that the diameter of the sample. Each experiment was performed in triplicate.

\section{Results and Discussion}

\subsection{Fabrication of chitosan mats}

Nanofibrous chitosan mats where prepared after electrospinning solutions of $3 \% \mathrm{w} / \mathrm{v}$ and $5 \% \mathrm{w} / \mathrm{v}$ of chitosan and according to the experimental design described above. The morphology of the mats was studied under the Scanning electron microscope and the results are grouped and summarized in the following figures.

Consistent viscosity measurements were not possible due to the solvent's volatility; hence the concentration is used instead as solution parameter.

\subsubsection{Effect of Polymer Concentration}


In Figures $1 \mathrm{~A}-1 \mathrm{~F}$, the nanofibre morphology obtained for the electrospun $5 \% \mathrm{w} / \mathrm{v}$ and $3 \% \mathrm{w} / \mathrm{v}$ solutions in a TFA/DCM solvent system at different combinations of applied voltage and humidity are presented.
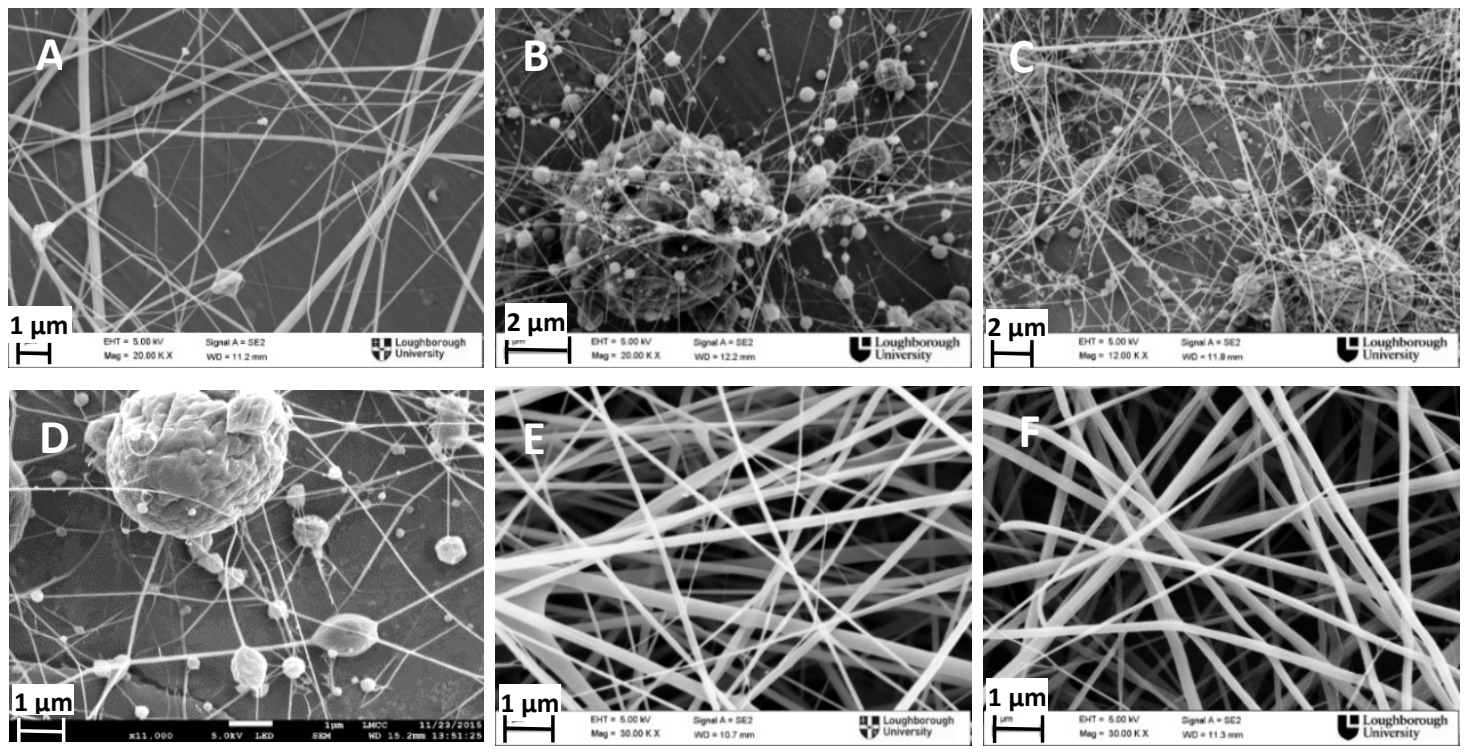

Figure 1: SEM pictures of chitosan solutions in TFA:DCM (80:20) of A: 5\%w/v and B: 3\% electrospun at $28.5 \mathrm{kV}$ and $45 \% \mathrm{RH}, \mathrm{C}: 5 \% \mathrm{w} / \mathrm{v}$ and D: $3 \% \mathrm{w} / \mathrm{v}$ electrospun at $23 \mathrm{kV}$ and $45 \% \mathrm{RH}, \mathrm{E}: 5 \% \mathrm{w} / \mathrm{v}$ and $\mathrm{F}: 3 \%$ electrospun at $28.5 \mathrm{kV}$ and $20 \% \mathrm{RH}$

As is shown in Figure 1, defect free nanofibre morphology is obtained at both concentrations when the applied voltage is high $(28.5 \mathrm{kV})$ and the relative humidity low $(20 \%)$. However, the solution with higher concentration of chitosan $(5 \% \mathrm{w} / \mathrm{v})$ seems to be more easily electrospun as it is shown in Figure 1A, where defect free nanofibre morphology is obtained for the 5\%w/v solution even at higher humidity, versus Figure 1B (3\%w/v) where mostly beads are formed when the same electrospinning parameters are used. This indicates that the $5 \% \mathrm{w} / \mathrm{v}$ solution is electrospinnable at a broader range of process parameters. This is expected, as, higher polymer concentration, generally results in more polymer chain entanglements (27) and higher solution viscosity and therefore in solutions that produce stable jets at a broader range of conditions, up to a threshold in concentration and viscosity, beyond which the viscosity of the solution is too high and electrospinning is not feasible. A comparison of the average diameters for the two solutions at the same electrospinning conditions, in Table 3, shows that higher chitosan concentration (5\%) leads to the formation of larger fibre diameters, for example increasing the chitosan concentration from $3 \%$ to $5 \%$, at $22.5 \mathrm{KV}$ and $20 \% \mathrm{RH}$, led to an increase in the fibre diameter from 94 to 104 $\mathrm{nm}$. This trend of increasing fibre diameter for increasing polymer concentration was observed for all the sets of electrospinning conditions, in accordance with the literature, due to the viscosity increase of the solution (20). Also, humidity seems to have a greater effect on the $3 \% \mathrm{w} / \mathrm{v}$ solution rather than the $5 \% \mathrm{w} / \mathrm{v}$. The surrounding water vapors generally remove surface charges from the jet. In the case of the $3 \% \mathrm{w} / \mathrm{v}$ solution, the consequence of charge removal seems to be more pronounced, resulting in domination of the elasticity effect over surface charge repulsion which is causing the jet to break up and form a bead on sting morphology. When the voltage is increased at high humidity, the increase of the electric field is counteracting the removal of charges from the jet and smooth nanofibers are obtained again (Fig. 1D). Furthermore, the fact that chitosan is a cationic polyelectrolyte should be taken into account. Pure chitosan is electrospinnable in TFA, as opposed to other acids, because it forms stable salts in TFA, prohibiting very strong repulsions between the chitosan chains. With the increase of surrounding humidity, however, this equilibrium is disrupted, because TFA being highly hygroscopic can straightly absorb water vapor from the surrounding. Therefore, increased charge repulsion between the chitosan chains may occur, while the jet is elongated and travelling towards the collector. At increased chitosan concentration, increased repulsion will occur, resulting in a more elongated jet, where the elastic effect causing the jet to contract is eliminated and thus smooth fibers can be obtained. These are the reasons why the effect of charge removal caused by increased humidity is dominant only at low voltage and low chitosan concentration. 


\subsubsection{Effect of process parameters (humidity \& voltage)}

In Figure 2, the nanofibre morphologies of $3 \% \mathrm{w} / \mathrm{v}$ and $5 \% \mathrm{w} / \mathrm{v}$ chitosan solutions in TFA/DCM, electrospun at different voltages and at values of high and low humidity are compared, in an attempt to examine if and how the relative humidity and the electric field affect the final nanofibre morphology.
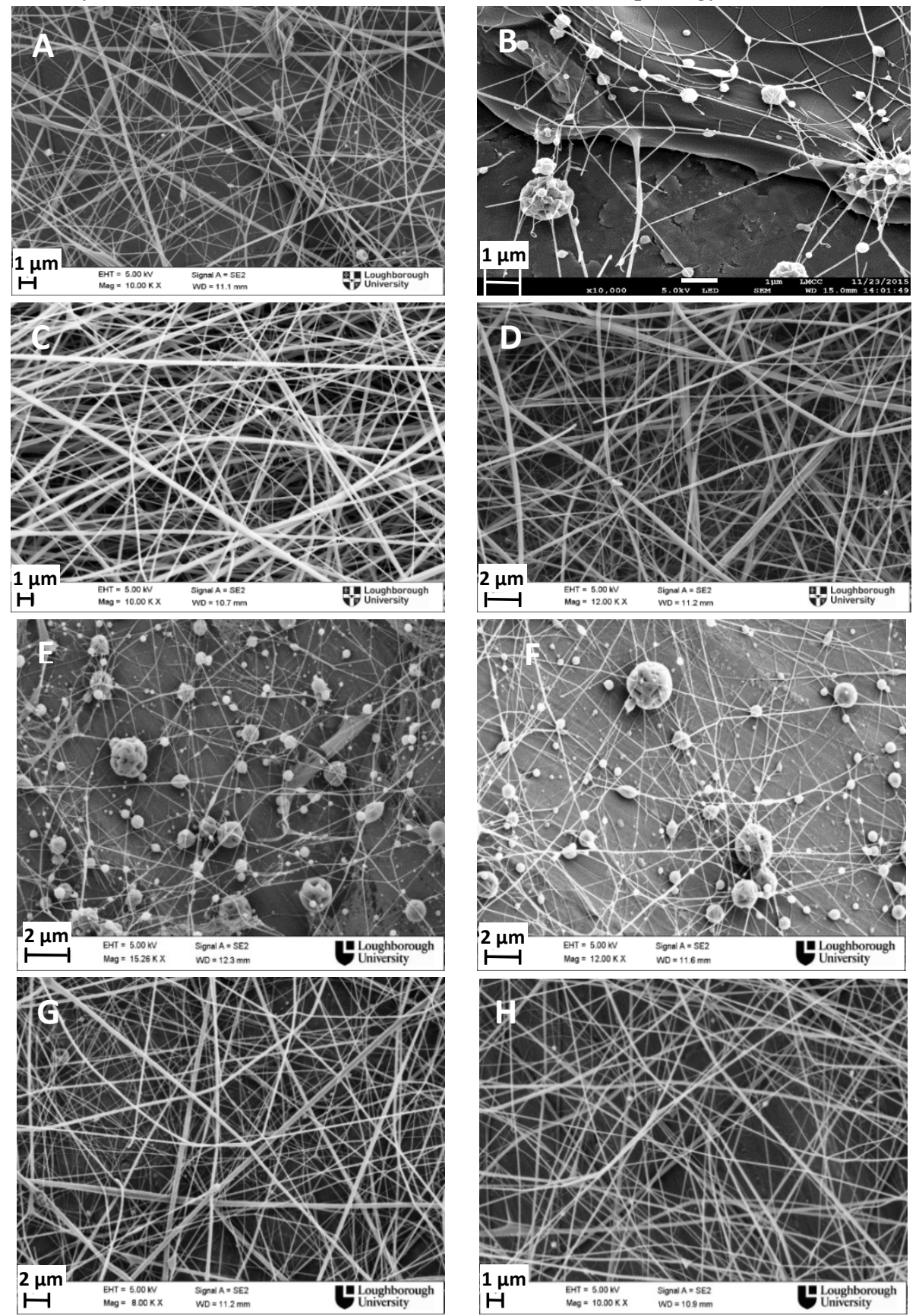

Figure 2: SEM pictures of chitosan solutions of $5 \% \mathrm{w} / \mathrm{v}$ electrospun at A: $28.5 \mathrm{kV}$ and $45 \% \mathrm{RH}, \mathrm{B}$ : $22.5 \mathrm{kV}$ and $45 \% \mathrm{RH}, \mathrm{C}: 28.5 \mathrm{kV}$ and $20 \% \mathrm{RH}, \mathrm{D}: 22.5 \mathrm{kV}$ and $20 \% \mathrm{RH}$ and $3 \% \mathrm{w} / \mathrm{v}$ solution electrospun at E: $28.5 \mathrm{kV}$ and $45 \% \mathrm{RH}, \mathrm{F}: 22.5 \mathrm{kV}$ and $45 \% \mathrm{RH}, \mathrm{G}: 28.5 \mathrm{kV}$ and $20 \% \mathrm{RH}, \mathrm{H}: 22.5 \mathrm{kV}$

As can be seen in Figure 2, high humidity appeared to have a negative impact on the electrospinnability of chitosan solutions when low voltage was applied for both solution concentrations (Figures $2 \mathrm{~B} \& \mathrm{~F}$ as opposed to Figures 2 D\&H respectively). Humidity seems to have an influence on the electrospinnability of chitosan, by removing charges from the jet, thus hindering the process. Also, higher humidity leads to slower solidification rate, as chitosan salt is hydrophilic and TFA is highly hygroscopic. As has been pointed out by Pelipenko et al. (28), when studying electrospinning of water based, or aqueous acetic acid solutions, at higher humidity values, since the solidification rate is lower, the jet surface gradually gets more concentrated as solvent evaporates and 
has the time to travel for longer (given adequate distance of the collector). This increase of concentration on the jet surface, and the increased travelling time due to slower solidification rate allows enough time for the elastic forces to overcome the plastic ones, causing the jet to contract and thus leading into gradual appearance of beads (28). It is also possible that absorbance of water vapors of the jet, due to the solvent's hygroscopicity, may be altering the solutions properties, i.e. increasing the interfacial tension and thus leading to the formation of beads.

Regarding the effect of the applied voltage, it becomes obvious that the voltage affects the electrospinnability of chitosan solutions, but it seems to be a secondary parameter when the solution concentration is low (3\%). For example, the morphology in Figure 2E does not differ from Figure $2 \mathrm{~F}$ where mostly beads are observed and Figure $2 \mathrm{G}$ does not differ from Figure $2 \mathrm{H}$, both showing uniform nanofibre morphology. This leads us to the conclusion that humidity is the governing parameter determining electrospinnability at low concentrations and the increase of the applied voltage doesn't seem to have any impact. On the contrary, for higher polymer concentration, the voltage seems to be a determining factor in order to improve nanofibre morphology from beaded fibres (Figure 2B) to homogenous defect free fibres (Figure 2A) at high relative humidity (RH). When low RH is used, both applied voltages yield good nanofibrous structure, but some broken fibres appear at high voltage (Figure 2D). This may be due to the fact that at increased voltage, the polymer jet undergoes extensive whipping and due to the low humidity, it may solidify before reaching the collector, thus the further whipping caused by the high electric field, may cause breakage of the solidified jet.

The slower solidification rate at high humidity that was commented on earlier, is also confirmed by the observation of smaller fibre diameters obtained at high humidity, as is shown in Table 3 . In the case of a hydrophobic polymer or a solvent immiscible with water, phase separation occurs resulting in rough nanofibre surface or pore formation would be more likely to be observed at high humidity.

Table 3: Average diameter \& coefficient of variation of chitosan fibres for high (50\%) and low (20\%) values of RH. The average diameter was calculated after counting the diameters of 200 nanofibres for each sample

\begin{tabular}{ccccc}
$\begin{array}{c}\text { Polymer Concentration } \\
(\mathrm{w} / \mathrm{v})\end{array}$ & $\begin{array}{c}\text { Applied } \\
\text { Voltage }(\mathrm{kV})\end{array}$ & $\begin{array}{c}\text { Relative } \\
\text { Humidity }\end{array}$ & $\begin{array}{c}\text { Average Diameter } \\
(\mathrm{nm})\end{array}$ & $\begin{array}{c}\text { Coefficient of } \\
\text { Variation }\end{array}$ \\
\hline $3 \%$, & 22.5 & $20 \%$ & 94 & 0.61 \\
$3 \%$ & 22.5 & $50 \%$ & - & - \\
\hline $3 \%$ & 26 & $50 \%$ & 85 & 0.83 \\
$3 \%$ & 26 & $20 \%$ & 96 & 0.89 \\
\hline $3 \%$ & 28.5 & $50 \%$ & - & - \\
$3 \%$ & 28.5 & $20 \%$ & 149 & 0.78 \\
\hline $5 \%$ & 22.5 & $50 \%$ & - & - \\
$5 \%$ & 22.5 & $20 \%$ & 104 & 0.46 \\
\hline $5 \%$ & 26 & $50 \%$ & 148 & 0.62 \\
$5 \%$ & 26 & $20 \%$ & 111 & 0.67 \\
\hline $5 \%$ & 28.5 & $50 \%$ & 149 & 0.52 \\
$5 \%$ & 28.5 & $20 \%$ & 163 & 0.65 \\
\hline
\end{tabular}

In Table 3, the way the humidity influences the fibre diameter is evident. Some nanofiber diameter data are missing, as at lower voltage $(22.5 \mathrm{kV})$ production of defect free nanofibers was not always successful and therefore direct comparison cannot be performed. For both solution concentrations, when the humidity is increased whilst all the other electrospinning parameters, including the applied voltage, are maintained the same, the average diameter drops, in accordance with observations of others in the literature $(28,29)$. For hydrophilic solutions such 
as this, at low humidity, the fast solvent evaporation causes the jet to solidify before having undergone adequate whipping resulting in larger diameter fibres. At high humidity, and given adequate tip to collector distance, the jet undergoes extensive whipping, having adequate time to solidify before reaching the collector. However, this phenomenon seems to be more pronounced in the case of the solution with the higher polymer concentration, for both applied voltages. As the solution is more concentrated, the solution viscosity increases and thus, the jet diameter is expected to be larger. The observation of the opposite trend is an indication that the polycationic nature of the chitosan affects the process in the opposite way. Increased chitosan concentration leads to extensive repulsion of surface charges, in the same way that the presence of inorganic salts is known to affect the electrospinning process and thus resulting in more whipping for the more concentrated solution when compared to the less concentrated one.

Comparing the electrospinning windows for the two different concentrations, as a general conclusion it can be said that both solutions (of different concentrations), are electrospinnable under the conditions examined with the experimental design and result in similar fibre morphology when same conditions are applied. One only exception is observed at high voltage and high RH, where electrospinning of the more concentrated solution (5\%) results in nanofibres with minor defects (Figures $2 \mathrm{~A}, \mathrm{~B}, \mathrm{C} \& \mathrm{D}$ ), while the $3 \%$ solution results in a string on bead morphology, where mostly beads of various sizes are formed during the process (Figures 2 E,F,G\&H), because of more chain entanglements that the higher concentration allows for.

Lastly, in Table 3 it is also shown that the electrospinning of 5\% solution results in thicker nanofibres for the same conditions, when compared to the $3 \%$ solution. The polymer concentration directly affects the size of the produced nanofibres (30), as more viscous solutions result in the formation of thicker fibres. It is worth noting here that all the counted diameters fall in the nanoscale. For the intended applications, nanofibres within a range similar to the collagen fibrils of the extracellular matrix, i.e. $10-500 \mathrm{~nm}$ would be advantageous (31). As for the coefficient of variation, the nanofibrous mats produced by use of $5 \%$ solution seem to be slightly more uniform in terms of nanofibre diameters than when a $3 \% \mathrm{w} / \mathrm{v}$ solution is used. For those reasons, the $5 \% \mathrm{w} / \mathrm{v}$ chitosan concentration will be used for further experiments, where PANI is incorporated in the blend.

\subsection{Production of PANI/CH blend mats}

The conductivity of the PANI/CH blends was measured for all blends just before electrospinning and is presented in Table 4.

Table 4: Measured Conductivity of the electrospinning blends

\begin{tabular}{lll} 
Blend PANI:CH ratio & Conductivity $(\mu \mathrm{S})$ & Temperature $\left({ }^{0} \mathrm{C}\right)$ \\
\hline \hline Pure Chitosan & 215 & 23 \\
\hline $1: 3$ & 278 & 21.8 \\
\hline $3: 5$ & 355 & 22.3 \\
\hline $1: 1$ & 430 & 20.7 \\
\hline
\end{tabular}

As is expected, the conductivity of the solution increases as the PANI content in the blend increases. These measurements were used in conjunction with the electrospinning windows of these blends. In Figures $3 \mathrm{~A}, \mathrm{~B} \& \mathrm{C}$ the contour and surface plots of the electrospinnability of PANI/CH blends of different ratios is shown plotted in relation to the applied voltage. 
A
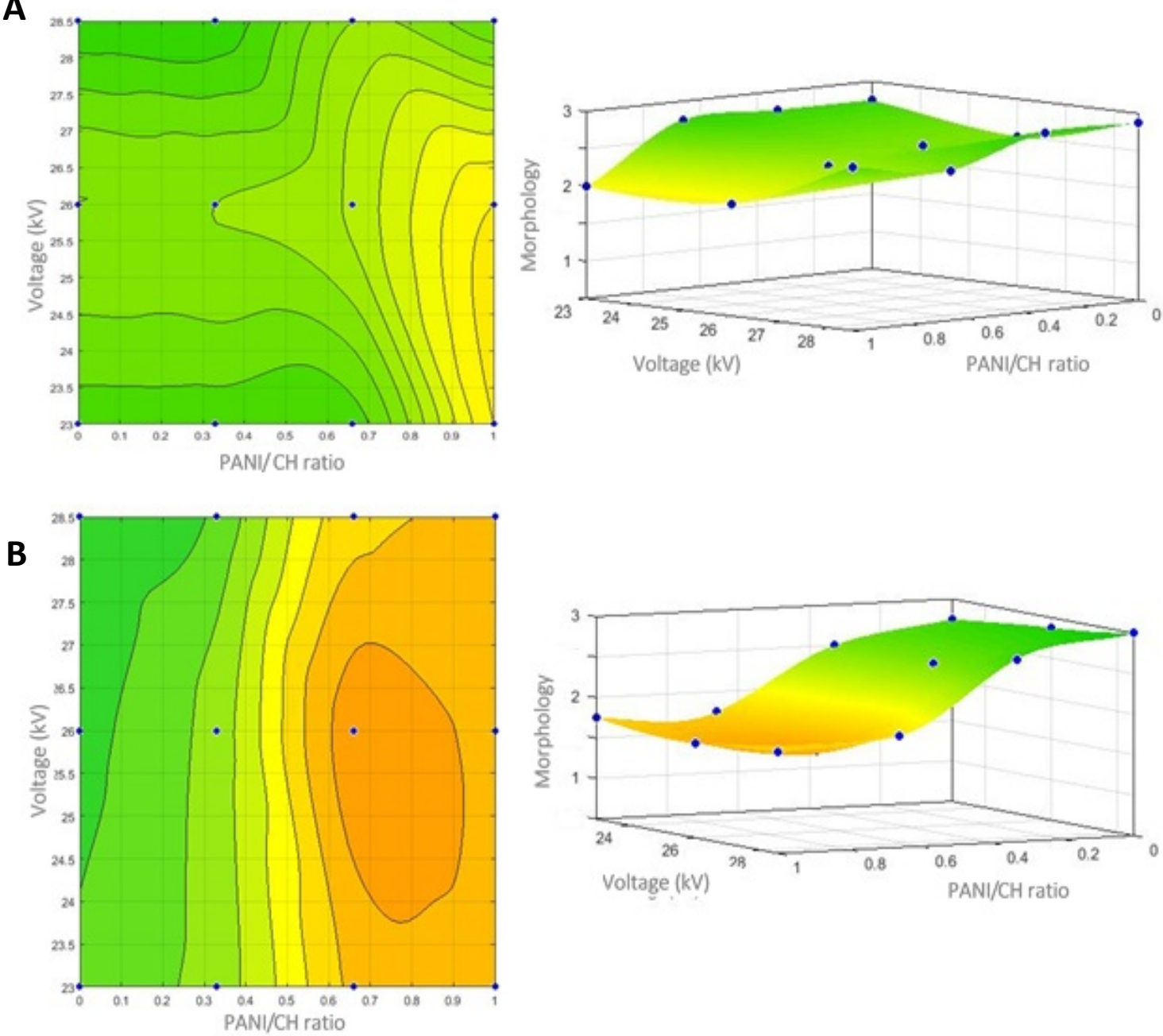

C
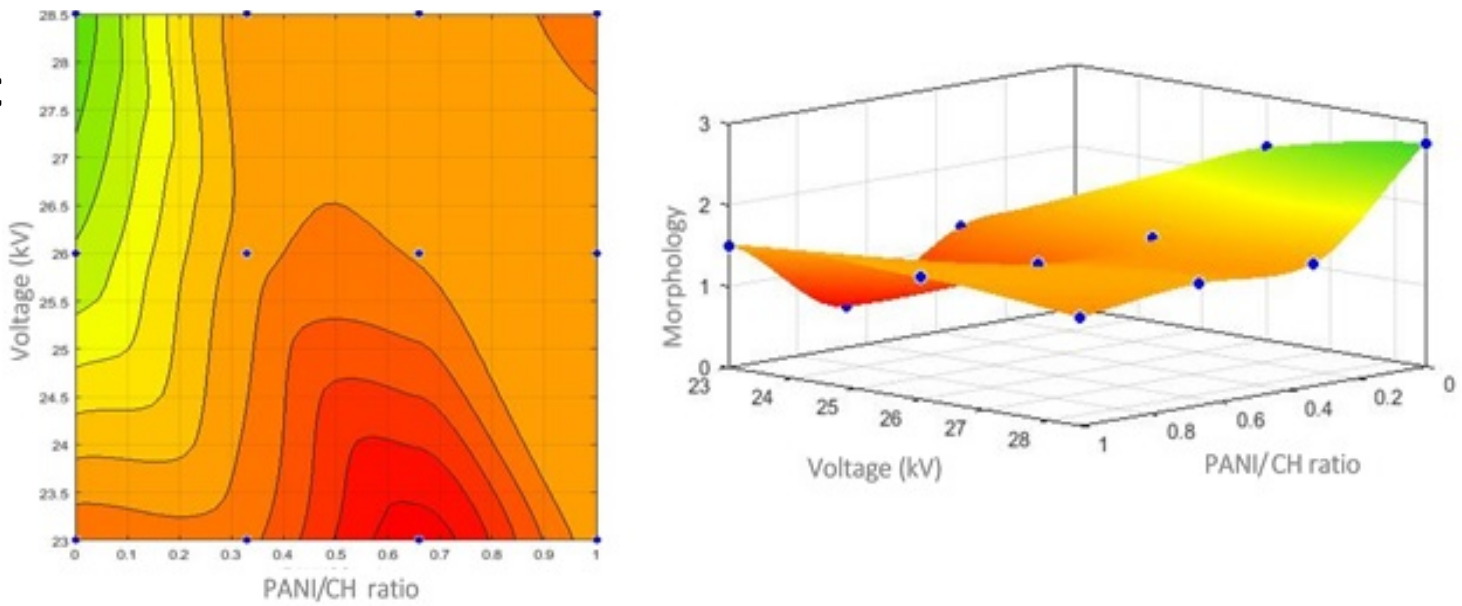

Figure 3: A, B \& C: Electrospinnability window of blends with different $\mathrm{PANI} / \mathrm{CH}$ ratios, at various voltages and at fixed RH: A: $20 \%$, B: $35 \%$, C: $50 \%$.

The morphology of the fibres was categorized in four groups and colour coding was used for the visualization of the electrospinning regimes in figures 3 and 4 as follows:

0 (red color): electrospinning was not possible/no fibres collected,

1 (orange color): electrospinning was possible with a lot of defects - string on bead morphology, wet mat 2 (yellow color): electrospinning was possible but with minor defects - bead on string morphology, broken fibres 3 (green color): defect free nanofibre morphology 
The gradual transitions from one category to the other are represented by incremental transitions (automatically generated by matlab) between these 4 main colours. For example, all the intermediate shades from red to orange represent the conditions where, mainly bead morphology prevailed over fibres. All the intermediate shades of orange to yellow, represent the conditions where fibre morphology prevailed over beads. Finally, all the intermediate shades from yellow to green, represent those conditions where any beads or other defects gradually disappeared and defect free fibres were formed.
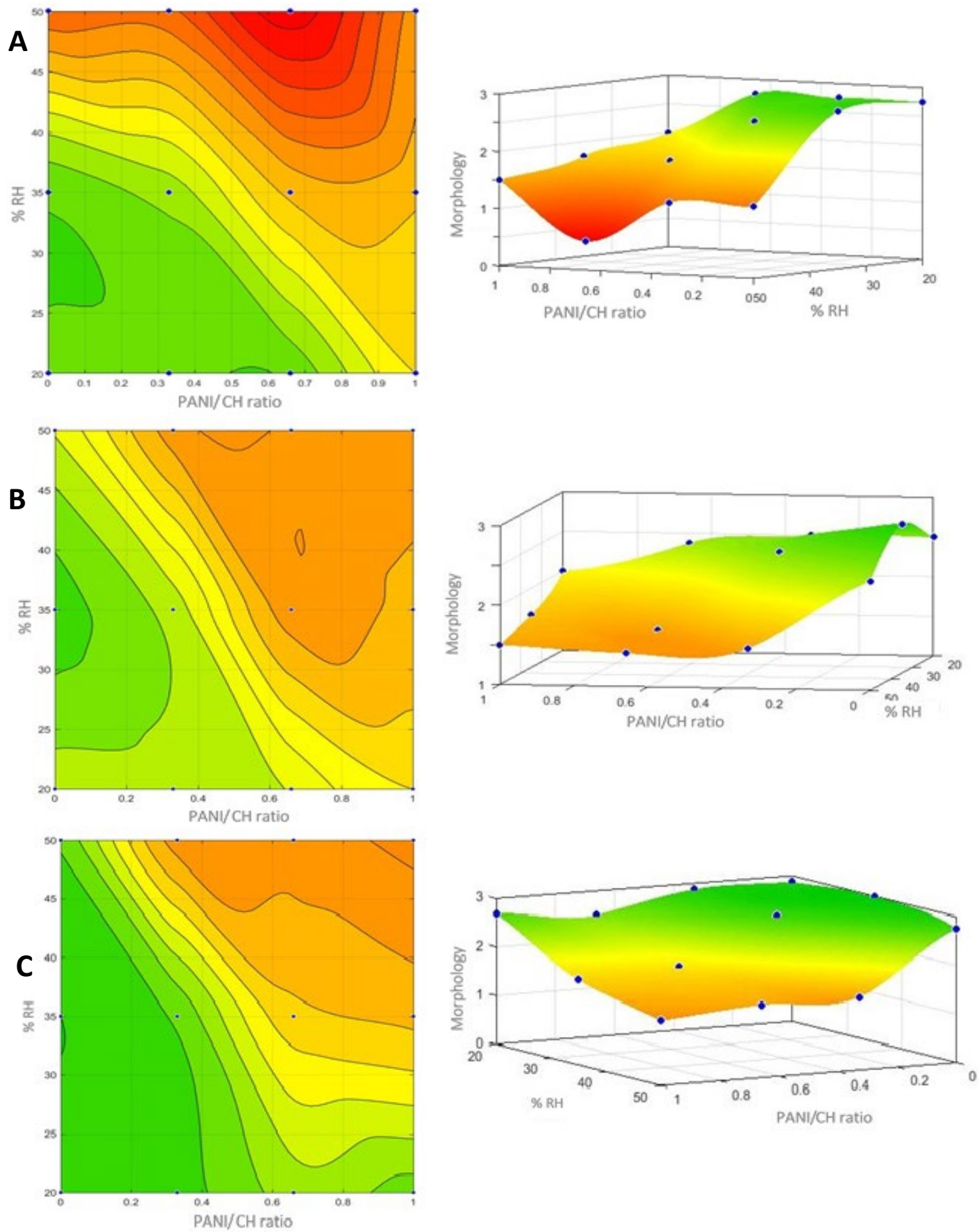

Figure 4 A, B \& C: Electrospinnability window of blends with different PANI/CH ratios, at various RH and at fixed applied voltage: A: $22.5 \mathrm{kV}, \mathrm{B}: 25.5 \mathrm{kV}, \mathrm{C}: 28.5 \mathrm{kV}$ 
As is shown in Figure 4, electrospinning becomes increasingly hindered as the RH increases. At $20 \%$ RH (Figure $3 \mathrm{~A}$ ), defect free nanofibres are produced for all the blends. Some beads appear only at high PANI/CH ratio and low applied voltage. As the RH increases, it is observed that the electrospinning becomes less and less successful with the higher PANI ratio blends being more influenced by the RH increase. At high RH (50\%) (Figure 3C), the only defect free nanofibres are obtained by electrospinning chitosan solutions without any PANI, highlighting the importance of the environmental humidity when electrospinning solutions of conducting polymers. It should also be noted that increasing the PANI ratio in the blend, the electrospinning window is being narrowed, highlighting the importance of control over the environmental parameters when electrospinning highly conductive blends.

Comparing Figures 4A, B and C contour and surface plots, it is obvious that the applied voltage affects the electrospinnability of PANI/CH blends. In general, as the applied voltage is increased, the electrospinning process is facilitated, especially for the PANI/CH blends with high PANI ratio, where defect free electrospun fibres are only obtained at high voltage and only for low values of relative humidity. These results can be compared directly with the study (32) conducted on the electrospinnability of a PANI blend with the organic polymer PEO which is not a polyelectrolyte. Humidity and voltage were found to impact the electrospinnability of the 50:50 ratio blend that was used in that specific study in a similar way, confirming that the effect is mainly due to the addition of the conducting polymer.

In Figure 5, the diameter distributions of the nanofibres produced from electrospinning PANI/CH blends at 20\% RH are presented. Figure 5 provides some interesting observations regarding the diameter distribution of $\mathrm{PANI} / \mathrm{CH}$ blends when electrospun at $20 \% \mathrm{RH}$. Horizontally, the diameter distributions for each one of the different blend ratios (PANI:CH 0:1, 1:3, 3:5 and 1:1) are depicted, when electrospun at different voltages. Vertically the effect of the different PANI ratio, is shown when the same voltage is applied (either $28.5 \mathrm{kV}, 26 \mathrm{kV}$, or $23 \mathrm{kV}$ ).

Firstly, it is obvious that the incorporation of PANI in the blend results in thinner diameters, as the count of nanofibres up to $100 \mathrm{~nm}$ increases, and the count of the larger ones decreases evidently, when comparing the 0 ratio blends (no PANI), to the rest of the blends, for all the applied voltages. This is depicted on the average diameter as well, which was lower for the solutions that incorporate PANI when compared to the pure chitosan one ( 0 ratio). Notably, that higher voltage results in thinner nanofibres for the blend containing the bigger ratio of PANI, whilst for the other blends, the average nanofibre diameter only fluctuates slightly for each voltage level but does not change significantly. In the case of the pure chitosan solution, the exact opposite trend is observed, meaning that the average diameter is evidently larger for higher voltage and decreases with decreasing voltage. This observation agrees with the observations by Zhang and colleagues, and Meechaisue et al., who all attributed this phenomenon to the solution being removed from the capillary tip faster at higher voltages, increasing the polymer's drawing rate $(33,34)$. However, this interpretation alone cannot explain the contradicting behavior of pure chitosan and PANI/CH solutions observed in this work. Another phenomenon attributing to the occurrence of larger diameters needs to be considered, the solvent evaporation rate. At higher voltages, the temperature of the jet may rise due to Joule heating effects, leading to faster solvent evaporation, hence faster jet solidification and less whipping occurs leading to the formation of larger diameters. The discrepancy between the electrospun solutions behavior regarding the way the applied voltage affects the average nanofibre diameter, indicates that there are competing phenomena taking place. Apart from the increase of flow rate, and faster solidification rate that are driving towards the formation of fibres with larger diameters, there is also the electrostatic repulsion within the polymer jet leading towards the formation of fibres with smaller diameters. When a conducting polymer is introduced in the solution, it seems to have a significant effect on this electrostatic repulsion, counteracting the increase on the flow and the solidification rate. Furthermore, as the PANI ratio increases in the blend, the total polymer concentration increases as well, which would be expected to lead to larger diameters, but this as well seems to be counteracted by the strong electrostatic repulsions, and the resulting extensive whipping, resulting in smaller diameters and highlighting the influence of PANI in the blend. 


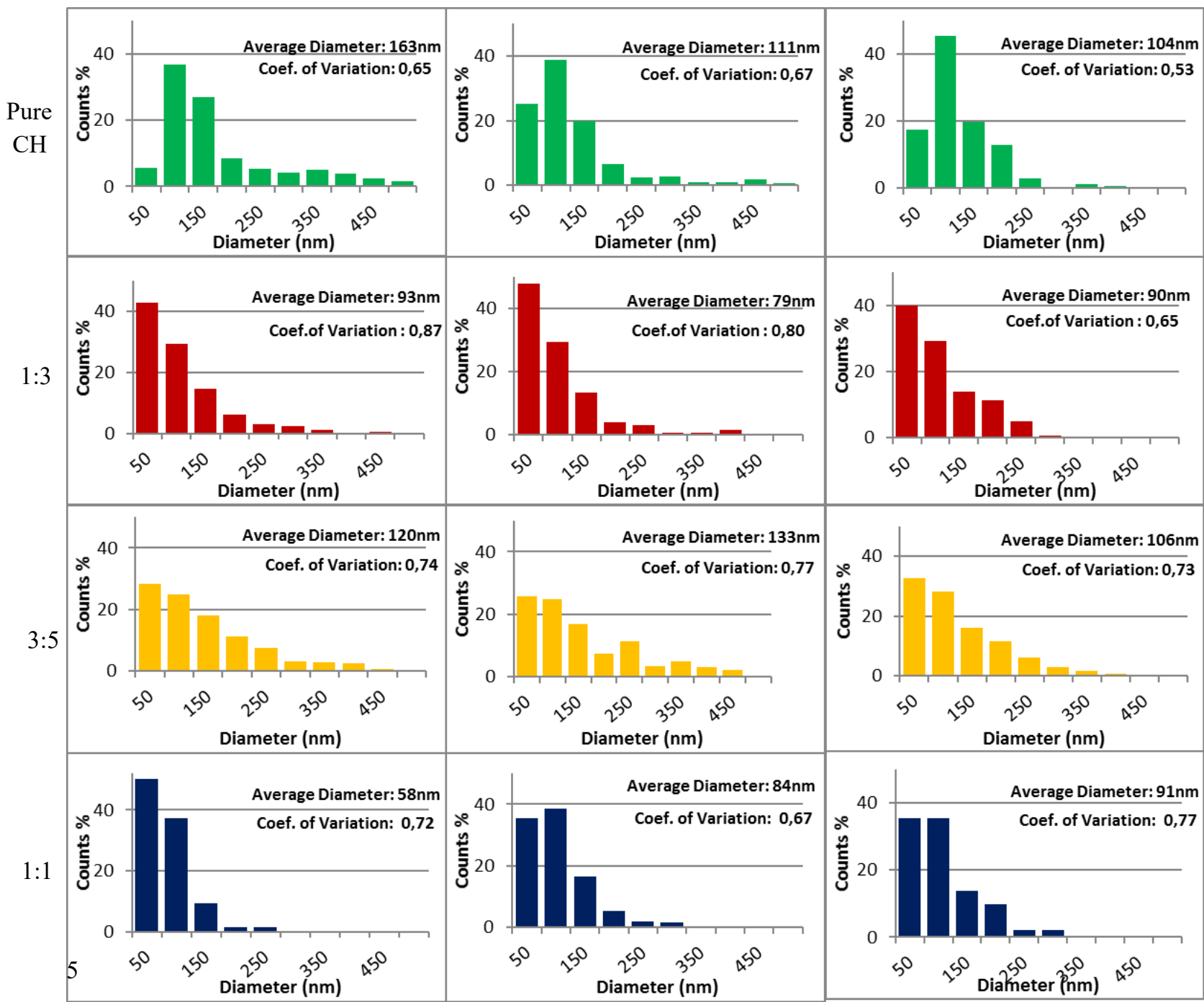

Figure 5: Diameter Distribution of PANI/CH blends electrospun at various voltages and at fixed $\mathrm{RH} 20 \%$

Finally, the distribution range, is obviously broader when electrospinning at $28.5 \mathrm{kV}$ rather than at $26 \mathrm{kV}$ or $22.5 \mathrm{kV}$, especially regarding the solutions with lower PANI contents. In order to explain that, the polarity of the chitosan itself must be taken into account too. Chitosan is a positively charged molecule and it has been reported that when electrospun under positive high voltage (like in the present study) multiple jets are formed, instead of one, indicating process instability (35) and thus leading to greater diameter distribution range. The addition of PANI in this study, seems to be counteracting this phenomenon. This happens probably not because of the PANI itself, but due to the dopant acid used, in this case CSA. The higher content of CSA in the solution seems to have an effect on the positively charged chitosan molecule. It has been shown that sulfonic acids act as hydrogen donors to the nitrogen atom in between the phenyl rings of the PANI molecule, forming hydrogen bonds (36). Moreover, the possibility of a single CSA molecule bonding two PANI chains by double hydrogen bonds has been shown to be high (37), leaving thus the sulfonate group negatively charged and possibly counterbalancing the charge of the chitosan molecule which is known to be positively charged under acidic conditions, preventing in that manner excessive charge repulsions and thus stabilizing the jet and resulting in more homogeneous distribution of nanofibre diameters. Indeed it has been reported that in polyelectrolyte solutions the ratio of the -COOH group of the polyanion to the $-\mathrm{NH}_{3}$ group of the polycation affects the electrospinnability, leading to decreased electrospinnability when it is above 1 (38). In this case, instead of $-\mathrm{COOH}$ group there is the $-\mathrm{S}(=\mathrm{O}) 2-\mathrm{OH}$ 
(sulfonyl hydroxide) group of the CSA but the same principle can be applied in order to explain the electrospinning instability occurring at higher chitosan ratios.

\subsection{Evaluation of Antibacterial Properties}

The antibacterial properties of the membranes were evaluated for their bactericidal efficiency against a model gram positive (B. subtilis) and a gram negative (E. coli) bacterial strain, in terms of their PANI:CH ratio (1:1, 3:5, 1:3 \& pure $\mathrm{CH}$ ) and their post-spinning treatment which is required to waterproof chitosan by rendering it insoluble. Two methods were used to waterproof the PANI membranes, neutralization with $\mathrm{NaOH}$, and crosslinking with glutaraldehyde vapor, as described in our previous paper (4). The difference between the two methods, is that the neutralization with $\mathrm{NaOH}$ deprotonates the amino groups of both the chitosan and the PANI molecule, reducing the conductivity of the blend, while the latter waterproofs the chitosan without deprotonating the amino groups of chitosan and PANI molecules, hence maintaining the blend conductivity. It must be noted that the untreated membranes, could not be thoroughly washed with PBS, as they would dissolve almost straight away. Therefore, these membranes are more likely to contain traces of toxic agents (such as solvents like TFA used during spinning) that could interfere with the antibacterial tests. A few representative photos of the inhibition zones are shown in Figure 6, where the inhibition zones are pointed with red arrows.
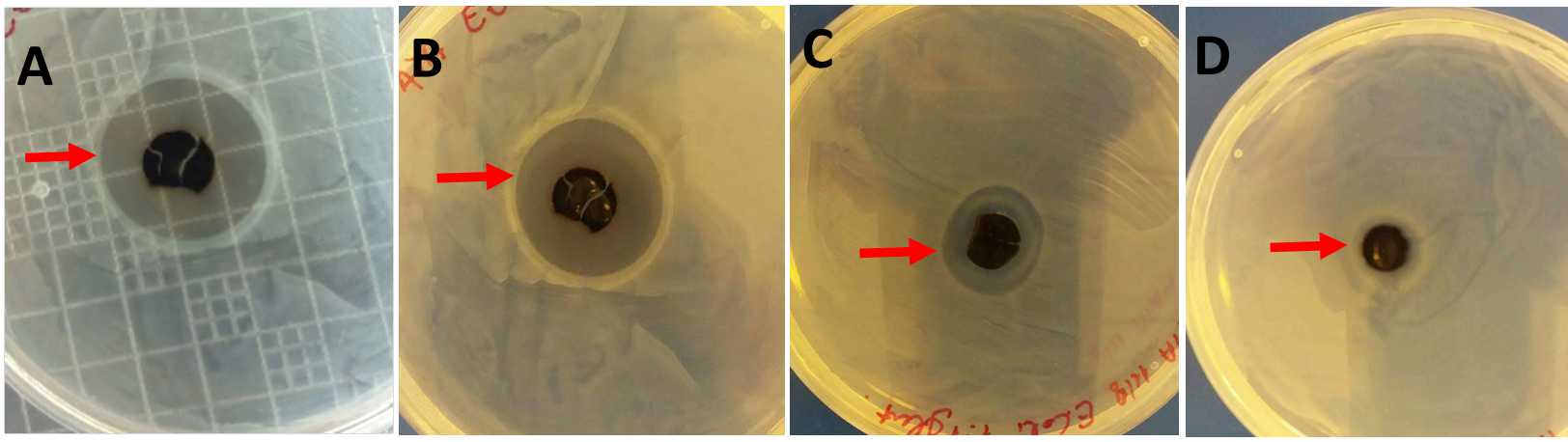

Figure 6: A: Untreated 1:1 PANI/CH against B. subtilis, B: Untreated 1:1 PANI/CH against E. coli, C: 1:1 $\mathrm{PANI} / \mathrm{CH}$ treated with glutaraldehyde against $E$. coli, D: neutralized 1:1 $\mathrm{PANI} / \mathrm{CH}$ against $E$. coli

A direct comparison was feasible for three types of membranes: untreated (no post spinning treatment) which are conductive but may contain traces of toxic agents; crosslinked with glutaraldehyde which are conductive, and neutralized, which have low to zero conductivity depending on the amount of PANI in the blend. More specifically, 1:1 samples of neutralized membranes would maintain higher conductivity compared to the 1:3 samples due to their higher content of PANI. 
The results from the inhibition zone test against $E$. coli and B. subtilis for neutralized with $\mathrm{NaOH}$, cross linked with glutaraldehyde and untreated membranes at different PANI/CH ratios are shown in Figures 7 A \& B. All bars represent mean values from triplicate experiments and the error bars represent the range of the measured values.

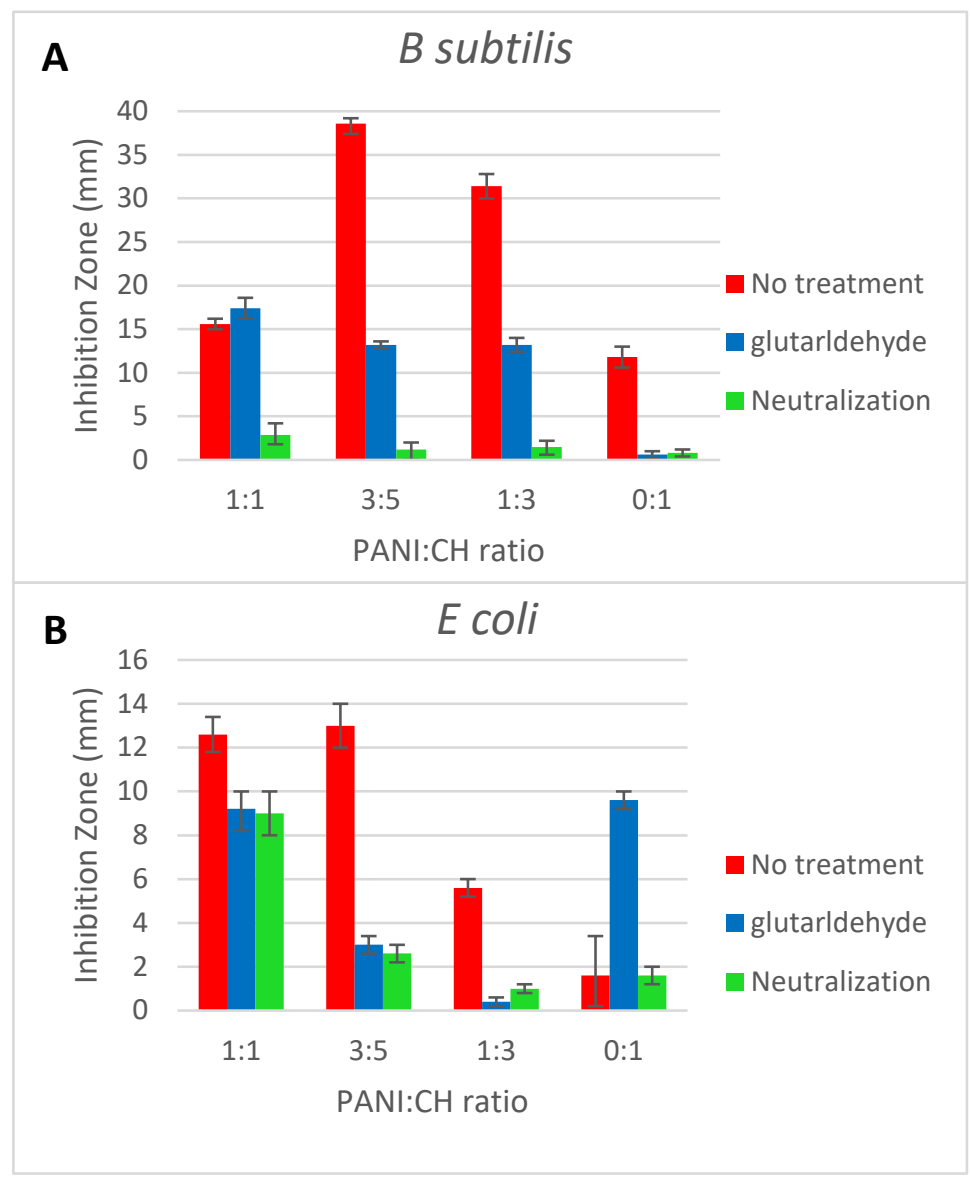

Figure 7: Antibacterial activity as evaluated by the inhibition zone (in mm) of blend mats against A: B. subtilis and B: E. coli. The reported inhibition diameters do not include the diameters of the mats.

A straight forward observation from Figure 7 is that for both model bacteria, the untreated mats exhibited greater inhibition zones than the crosslinked and the neutralized ones. This indicates, that remaining traces of toxic solvents, such as TFA, may be contributing to an increase in the total antibacterial activity of the untreated membranes. Each TFA molecule contains three Fluorine atoms, that have shown bactericidal activity (39), and may interact with the bacterial strains used in this study, enhancing the total antibacterial efficacy of the untreated membranes. Figure 7A shows a decrease of the inhibition zone for the untreated 1:1 PANI/CH samples compared to the other blends. Moreover, the inhibition zone of the 1:1 untreated samples is almost equal to the inhibition zone of the 1:1 PANI/CH crosslinked samples, indicating equal bactericidal efficacy. This supports the suggestion that the untreated 1:1 samples did not contain any toxic agents, and that their bactericidal activity represents the real one of the conductive polymer membrane. It should be noted that any toxic agents only enhanced the inhibition effects, and that the inhibition zones of the treated membranes reported in this study agree with the inhibition zones of other antimicrobial agents reported in the literature (26). For example, Carami et al. incorporated thymol in their PCL electrospun mats achieving inhibition zones of $7.8 \mathrm{~mm}$ against E. Coli, which also translated in accelerated wound healing in vivo (41). Generally the inhibition zones reported in this study, are also in agreement with the reported inhibitions zones obtained from antibiotics and other materials with antibacterial properties such as silver nanoparticles $(42,43)$. Although in vivo studies were out of the scope of our study, the inhibition zones that were observed here (the ones $>10 \mathrm{~mm}$ ) seem promising for further in vivo testing. 
A second observation is that the crosslinked membranes, which maintain the protonated states of PANI and chitosan, and hence the conductivity of the membrane, form a broader inhibition zone than the neutralized ones. These results agree with the studies suggesting that the cationic nature of the protonated state of chitosan, as well as the protonated state of PANI salt, may be affecting the bacteria's cell membrane $(13,15)$. It has been shown that chitosan in its neutral form, where the amine groups are deprotonated exhibits no antibacterial properties when tested against a variety of bacterial strains. The lack of interaction with the bacterial cell walls in this case, apart from the lack of charge of the amino groups can also be attributed to the poor solubility that chitosan presents in non-acidic $\mathrm{pH}(11)$.

Comparing Figures 7A and 7B, it can be observed that the antibacterial activity is stronger against B. subtilis which is gram positive than $E$. coli which is gram negative. A similar trend was observed by Goy et al (39), who reported a higher antimicrobial activity of chitosan against Gram-positive $S$. aureus as compared to E. coli. Gramnegative bacteria are generally considered more resistant to antibiotics because of an extra outer cell membrane that they possess (40), which is most probably the reason why they seem to be less affected by the inhibitory effect of the mats tested in this study. It can also be observed that the mat with the higher PANI/CH ratio, retains high antibacterial activity against $E$. coli even after neutralization, suggesting that the high PANI content contributes greatly to the inhibitory role, even when conductivity is partially lost. This can be directly compared with the zero content mat, where similar inhibitory effect is exhibited when glutaraldehyde is used as cross-linker, while this is almost lost when chitosan is deprotonated, indicating that the inhibitory effect is mainly due to the effect of PANI. Gizdavic - Nikolaidis et al., have shown that polyanilines functionalized with $-\mathrm{OCH}_{3},-\mathrm{CH}_{3},-\mathrm{SO}_{3} \mathrm{H}$ and $-\mathrm{Cl}$ substituents seem to share the same bactericidal effect with particular cationic antimicrobial peptides (AMP), by interacting with and inserting into anionic bacterial membranes, thereby compromising membrane integrity and cell division, leading to cell lysis and death (16). They also showed that exposure to a $-\mathrm{SO}_{3} \mathrm{H}$ functionalized PANI, led to significant changes in the expression levels of $218(5.1 \%)$ genes which are amongst others involved in biofilm formation, energy metabolism and protection from oxidative stress, targeting simultaneously several intracellular activities which could minimize the chances for the development of microbial resistance. In their particular study however, the antibacterial activity was assessed using suspension of the functionalized PANI's in broth whereas here, the bactericidal activity of PANI is assessed in a ready to use membrane form. Functionalized PANIs are known for better solubility in comparison to pure PANI, allowing for more interaction with the bacterial walls, and this might be a significant factor contributing to their higher bactericidal efficiency when compared to pure PANI. Additionally, functionalized PANI usually exhibits lower conductivities than PANI and in most cases it cannot be electrospun without the presence of a carrier polymer. Therefore, nanofibrous mats from functionalized PANI's might not exhibit as good bactericidal activity as their respective suspensions. In this current study, the aim was to assess the bactericidal activity of the nanofibrous membrane, which is the form in which PANI could be used in a wound dressing, in order to obtain results that are more clinically relevant.

Neutralized mats are not efficient against B. subtilis, but cross-linked ones, and especially the one with high PANI content exhibit good bactericidal activity indicating that retention of the protonated state and conductivity of PANI is absolutely necessary for the interaction of the mat with the bacteria's cell membrane, in order to exhibit bactericidal activity. Regarding the PANI:CH ratio, it is shown that especially in the case of E. coli, the higher PANI ratio increases the membranes' antimicrobial activity, with the two membranes containing the higher PANI concentrations $(1: 1 \& 3: 5)$ performing significantly better $(\mathrm{p}<0.01)$ than the ones with lower PANI concentrations $(1: 3 \&$ pure $\mathrm{CH})$, in the case of untreated and neutralized mats. In the case of $B$. subtilis all membranes containing PANI exhibit similar antimicrobial activity but a synergistic effect between PANI and chitosan is observed in 3:5 and 1:3 ratios in untreated membranes. Regarding the pure chitosan mats, in the case of B. subtilis it seems that the different cross-linking method, thus the protonated vs the deprotonated state has no effect on bactericidal activity, with both of them being very low, thus leading to the conclusion that indeed the soluble state of chitosan is determining for its bactericidal effect. In the case of E. coli, on the contrary, the glutaraldehyde cross-linked chitosan seems to retain some of its bactericidal activity when compared to the completely deprotonated molecule. This highlights the need for further study into the intracellular mechanisms making these two bacterial strains reacting so differently to the presence of chitosan. 


\section{Conclusions}

An extensive study has been conducted on the electrospinning of chitosan and of PANI/CH solutions. Chitosan can be electrospun successfully when diluted in a TFA:DCM (80:20) solvent system, at a concentration of 5\% $\mathrm{w} / \mathrm{v}$ and at several combinations of electrospinning parameters (applied voltage, TCD, environmental humidity etc). Solutions made from blending chitosan and PANI at different ratios were successfully electrospun, as in this case the PANI and chitosan molecules can freely move and orientate when high voltage is applied, thus formation of a continuous jet is rendered possible. The TFA:DCM (80:20) solvent system was found to be suitable for the electrospinning not only of chitosan solutions, which was expected, but for PANI solutions too, as it was found to dissolve CSA doped PANI very well. It was found that applied voltage is the dominant factor affecting the electrospinning of such solutions and as the content of PANI in the blend increases, humidity becomes an important parameter affecting electrospinnability. As for the effect of the incorporation of PANI in the blend, on the diameter of the produced nanofibres, it was observed that the incorporation of PANI in the blend results in thinner diameters. Higher applied voltage also generally results in higher counts of thinner nanofibres for the blends containing higher amount of PANI.

It has also been shown that blend $\mathrm{CH} / \mathrm{PANI}$ mats exhibit antibacterial activity, higher against Gram positive $B$. subtilis and lower against gram negative $E$. coli rendering them interesting candidates for the production of wound dressings. It has to be noted that higher PANI content mats did not need cross-linking to retain their integrity in aqueous environments, allowing for the use of the fully protonated state of chitosan in the blend which seems to be a more efficient anti-bacterial agent than its deprotonated state. For the mats with less or no PANI content, an alternative waterproofing method which consisted of crosslinking with the aid of glutaraldehyde vapors, proved to benefit the antibacterial action of these membranes. Finally, the membranes containing higher concentration of PANI seemed to be more efficient against both bacterial strains which is promising since the conducting properties introduced by means of PANI incorporation, can also potentially offer a tool for controlled release of bioactive substances and/or electrical excitation of cells in biomedical applications.

\section{References}

1. Punjabi PB, Chauhan NPS, Jangid NK, Juneja P. Conducting polymers: Biodegradable tissue engineering. Encycl Biomed Polym Polym Biomater. 2015;(April 2016):1972-81.

2. Katti DS, Robinson KW, Ko FK, Laurencin CT. Bioresorbable nanofiber-based systems for wound healing and drug delivery: Optimization of fabrication parameters. J Biomed Mater Res. 2004;70B(2):286-96.

3. Cargill M, Ireland JS, Bolk S, Roxbury W, Us MA, Mccarthy JJ. Dispensing device and method for forming material. Vol. 1, United States Patent. 6,252,129 B1, 2001.

4. Moutsatsou P, Coopman K, Georgiadou S. Biocompatibility assessment of conducting PANI/chitosan nanofibers for wound healing applications. Polymers (Basel). 2017;9(12).

5. Abrigo M, McArthur SL, Kingshott P. Electrospun nanofibers as dressings for chronic wound care: Advances, challenges, and future prospects. Macromol Biosci. 2014;14(6):772-92.

6. Huang Z, Zhang Y, Kotaki M, Ramakrishna S. A review on polymer nanofibers by electrospinning and their applications in nanocomposites. 2003;63:2223-53.

7. Gu BK, Park SJ, Kim MS, Kang CM, Kim J Il, Kim CH. Fabrication of sonicated chitosan nanofiber mat with enlarged porosity for use as hemostatic materials. Carbohydr Polym. 2013 Aug;97(1):65-73.

8. Croisier F, Jérôme C. Chitosan-based biomaterials for tissue engineering. Eur Polym J. 2013;49(4):780 92.

9. Marcasuzaa P, Reynaud S, Ehrenfeld F, Khoukh A, Desbrieres J. Chitosan-graft-polyaniline-based hydrogels: Elaboration and properties. Biomacromolecules. 2010;11(6):1684-91.

10. Jayakumar R, Prabaharan M, Reis RL, Mano JF. Graft copolymerized chitosan - Present status and applications. Carbohydr Polym. 2005;62(2):142-58.

11. Rabea EI, Badawy MET, Stevens C V., Smagghe G, Steurbaut W. Chitosan as antimicrobial agent: Applications and mode of action. Biomacromolecules. 2003;4(6):1457-65.

12. El Hadrami A, Adam LR, El Hadrami I, Daayf F. Chitosan in plant protection. Mar Drugs. 2010;8(4):968-87.

13. Helander IM, Nurmiaho-Lassila EL, Ahvenainen R, Rhoades J, Roller S. Chitosan disrupts the barrier properties of the outer membrane of Gram-negative bacteria. Int J Food Microbiol. 2001;71(2-3):23544.

14. Shiy N, Guo X, Hemin Jing, Gong Yang J, Sun C. Antibacterial effect of the conducting polyaniline. J 
Mater Sci Technol. 2006;22(3):289-90.

15. Kucekova Z, Kasparkova V, Humpolicek P, Sevcikova P, Stejskal J. Antibacterial properties of polyaniline-silver films. Chem Pap. 2013;67(8):1103-8.

16. Gizdavic-Nikolaidis MR, Bennett JR, Swift S, Easteal AJ, Ambrose M. Broad spectrum antimicrobial activity of functionalized polyanilines. Acta Biomater. 2011;7(12):4204-9.

17. Humpolicek P, Kasparkova V, Saha P, Stejskal J. Biocompatibility of polyaniline. Synth Met. 2012;162(7-8):722-7.

18. Ohkawa K, Cha D, Kim H, Nishida A, Yamamoto H. Electrospinning of Chitosan. Macromol Rapid Commun. 2004;25(18):1600-5.

19. Sun K, Li ZH. Preparations, properties and applications of chitosan based nanofibers fabricated by electrospinning. Express Polym Lett. 2011;5(4):342-61.

20. Casasola R, Thomas NL, Georgiadou S. Electrospinning of poly(lactic acid): Theoretical approach for the solvent selection to produce defect-free nanofibers. J Polym Sci Part B Polym Phys.

2016;54(15):1483-98.

21. Nizioł J, Gondek E, Plucinski KJ. Characterization of solution and solid state properties of polyaniline processed from trifluoroacetic acid. J Mater Sci Mater Electron. 2012;23(12):2194-201.

22. Palmer SJ. Effect of temperature on the surface tension. Phys Educ. 1976;11(2).

23. Torres-Giner S, Ocio MJ, Lagaron JM. Development of active antimicrobial fiber based chitosan polysaccharide nanostructures using electrospinning. Eng Life Sci. 2008;8(3):303-14.

24. Frohbergh ME, Katsman A, Botta GP, Lazarovici P, Schauer CL, Wegst UGK, et al. Electrospun hydroxyapatite-containing chitosan nanofibers crosslinked with genipin for bone tissue engineering. Biomaterials. 2012;33(36):9167-78.

25. Ma X, Ge J, Li Y, Guo B, Ma PX. Nanofibrous electroactive scaffolds from a chitosan-grafted-aniline tetramer by electrospinning for tissue engineering. RSC Adv. 2014;4(26):13652-61.

26. Dhivya C, Vandarkuzhali SAA, Radha N. Antimicrobial activities of nanostructured polyanilines doped with aromatic nitro compounds. Arab J Chem. 2015;

27. Sill TJ, von Recum HA. Electrospinning: Applications in drug delivery and tissue engineering. Biomaterials. 2008;29(13):1989-2006.

28. Pelipenko J, Kristl J, Janković B, Baumgartner S, Kocbek P. The impact of relative humidity during electrospinning on the morphology and mechanical properties of nanofibers. Int J Pharm. 2013 Nov;456(1):125-34.

29. De Vrieze S, Van Camp T, Nelvig A, Hagström B, Westbroek P, De Clerck K. The effect of temperature and humidity on electrospinning. J Mater Sci. 2009 Oct;44(5):1357-62.

30. Li W, Shanti RM, Tuan RS. Electrospinning technology for nanofibrous scaffolds in tissue engineering. CSSR K, editor. Nanotechnologies Life Sci. 2006;9(October 2015):135-87.

31. Zhang Y. Electrospun nanofibers with tunable electrical conductivity. 2013;30-8, 68. Available from: http://dspace.mit.edu/handle/1721.1/81690

32. Moutsatsou P, Coopman K, Smith MB, Georgiadou S. Conductive PANI fibers and determining factors for the electrospinning window. Polym (United Kingdom). 2015;77:143-51.

33. Zhang C, Yuan X. Study on morphology of electrospun poly (vinyl alcohol) mats. Eur Polym J. 2005;41:423-32.

34. Meechaisue C, Dubin R, Supaphol P, Hoven VP, Kohn J. Electrospun mat of tyrosine-derived polycarbonate fibers for potential use as tissue scaffolding material. J Biomater Sci Polym Ed. 2006;17(9):1039-56.

35. Terada D, Kobayashi H, Zhang K, Tiwari A, Yoshikawa C, Hanagata N. Transient charge-masking effect of applied voltage on electrospinning of pure chitosan nanofibers from aqueous solutions. Sci Technol Adv Mater. 2012 Jan;13(1):15003.

36. Foreman JP, Monkman AP. Theoretical investigations into the structural and electronic influences on the hydrogen bonding in doped polyaniline. Synth Met. 2003;135-136:375-6.

37. Łuzny W, Piwowarczyk K. Hydrogen bonds in camphorsulfonic acid doped polyaniline. Polimery/Polymers. 2011;56(9):652-6.

38. Ma G, Liu Y, Fang D, Chen J, Peng C, Fei X, et al. Hyaluronic acid/chitosan polyelectrolyte complexes nanofibers prepared by electrospinning. Mater Lett. 2012;74:78-80.

39. Ishihara M, Kosaka T, Nakamura T, Tsugawa K, Hasegawa M, Kokai F, et al. Antibacterial activity of fluorine incorporated DLC films. Diam Relat Mater. 2006;15(4-8):1011-4.

40. Goy RC, Morais STB, Assis OBG. Evaluation of the antimicrobial activity of chitosan and its quaternized derivative on E. Coli and S. aureus growth. Vol. 26, Brazilian Journal of Pharmacognosy. 2016. p. 122-7.

41. Salton MRJ, Kim K-S. Chapter 2: Structure. In: Baron S, editor. Medical Microbiology. 4th Editio. Galveston (TX): University of Texas Medical Branch at Galveston; 1996. 
42. Karami Z, Rezaeian I, Zahedi P, Abdollahi M. Preparation and performance evaluations of electrospun poly( $\varepsilon$ - caprolactone), poly(lactic acid), and their hybrid (50/50) nanofibrous mats containing thymol as an herbal drug for effective wound healing. J Appl Polym Sci. 2013;129(2):756-66.

43. Chowdhury NA, Al-Jumaily AM. Regenerated cellulose/polypyrrole/silver nanoparticles/ionic liquid composite films for potential wound healing applications. Wound Med. 2016;14:16-8.

44. Shahverdi AR, Fakhimi A, Shahverdi HR, Minaian S. Synthesis and effect of silver nanoparticles on the antibacterial activity of different antibiotics against Staphylococcus aureus and Escherichia coli. Nanomedicine Nanotechnology, Biol Med. 2007;3(2):168-71. 